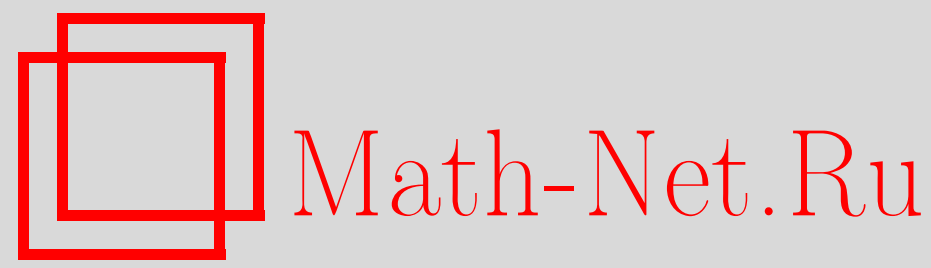

М. В. Йованович, Замечание о сильно выпуклых и квазивыпуклых функциях, Матем. заметки, 1996, том 60, выпуск 5, 778-779

DOI: https://doi.org/10.4213/mzm1892

Использование Общероссийского математического портала Math$\mathrm{Net.Ru} \mathrm{подразумевает,} \mathrm{что} \mathrm{вы} \mathrm{прочитали} \mathrm{и} \mathrm{согласны} \mathrm{с} \mathrm{пользователь-}$ ским соглашением

http://www.mathnet.ru/rus/agreement

Параметры загрузки:

IP : 35.173 .219 .12

26 апреля 2023 г., $07: 37: 18$

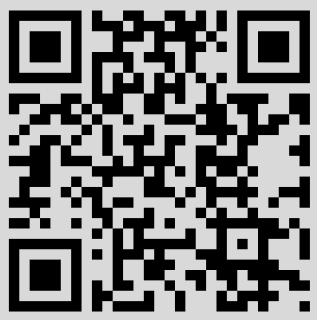




\section{ЗАМЕЧАНИЕ О СИЛЬНО ВЫПУКЛЫХ И КВАЗИВЫПУКЛЫХ ФУНКЦИЯХ}

\section{М.В. Йованович}

В настоящей заметке показано, как исследование сильной (квази) выпуклости функции многих переменных сводится к исследованию сильной (квази) выпуклости функции одной переменной.

Пусть $U \subseteq \mathbb{R}^{n}$ - непустое выпуклое множество, $J(u)$ - действительная функция, определенная на $U$. Обозначим

$$
\begin{aligned}
V_{J}(u, v ; \lambda) & =\lambda J(u)+(1-\lambda) J(v)-J(\lambda u+(1-\lambda) v), \\
Q_{J}(u, v ; \lambda) & =\max \{J(u), J(v)\}-J(\lambda u+(1-\lambda) v), \\
\tau(u, v ; \lambda) & =\lambda(1-\lambda)\|u-v\|^{2},
\end{aligned}
$$

где $u, v \in U, \lambda \in[0,1]$.

Как известно, функция $J(u)$ (квази) вьпукла на $U$, если $\left(Q_{J}(u, v ; \lambda) \geqslant 0\right)$ $V_{J}(u, v ; \lambda) \geqslant 0$ при всех $u, v \in U$, всех $\lambda \in[0,1]$.

Выпуклая функция, очевидно, квазивыпукла. Важные подклассы образуют сильно выпуклые (CB) и сильно квазивыпуклые функции (CKB) [1], [2]. Функция $J(u)$ назьвается сильно (квази) выпуклой на $U$, если существует постоянная $(s>0)$ $r>0$ такая, что для любых $u, v \in U, \lambda \in[0,1]$ выполняется

$$
\left(Q_{J}(u, v ; \lambda) \geqslant s \tau(u, v ; \lambda)\right) \quad V_{J}(u, v ; \lambda) \geqslant r \tau(u, v ; \lambda) .
$$

Класс CKВ функций является расширением класса СВ функций.

ПРИМЕР 1. Функция $g_{1}(t)=\sqrt{(t+a)^{2}+b-a^{2}}, a, b \in \mathbb{R}, b-a^{2}>0$, будет СВ на $[0, c], c>0$, с постоянной

$$
r=\frac{b-a^{2}}{2\left(\max \left\{g_{1}(0), g_{1}(c)\right\}\right)^{3}},
$$

так как $g_{1}^{\prime \prime}(t) \geqslant 2 r\left[3\right.$, c. 185]. Следовательно, $g_{1}(t)$ CКВ с постоянной $s=r$. Непосредственно проверяется, что $g_{1}(t)$ СКВ с постоянной

$$
s_{1}=\frac{1}{2 \max \left\{g_{1}(0), g_{1}(c)\right\}} \text {. }
$$

ПримеР 2. Функция $g_{2}(t)=|t+a| \mathrm{CKB}$ на $[0, c], c>0$, с постоянной $s_{2}=1 / c$, но не СB.

Как известно, $J(u)$ тогда и только тогда (квази) выпукла на $U \subseteq \mathbb{R}^{n}$, когда для произвольных фиксированных $u, v \in U$ функция $f(\lambda)=J(v+\lambda(u-v))$ (квази) выпукла на $[0,1]$.

В [3, с. 188] предлагается доказать, что функция $J(u)$ СВ на $U$ тогда и только тогда, когда $f(\lambda)$ является $\mathrm{CB}$ на $[0,1]$ с одной и той же для всех $u, v \in U$ постоянной сильной выпуклости. Это утверждение не имеет места. Так, например, функция $J(u)=\|u\|^{2} \mathrm{CB}$ на $\mathbb{R}^{n}$ с константой сильной выпуклости $r=1$, а функция $f(\lambda)=\|v+\lambda(u-v)\|^{2} \mathrm{CB}$ с константой $r=\|u-v\|^{2}$, зависящей от $u, v$.

Однако, нетрудно показать, что справедлива следующая 
ТЕОРема 1. Функиия $J(и)$ сильно (квази) выпукла на $U$ тогда и только тогда, когда функиия

$$
g(t)=J\left(v+\frac{t}{\|u-v\|}(u-v)\right)
$$

является сильно (квази) выпуклой на $[0,\|u-v\|]$ с одной и той жсе для всех $u, v \in U, u \neq v$, постоянной.

ТЕОРема 2. Евклидова норма $J(u)=\|u\|$ СКВ на всяком ограниченном випуклом множестве $U \subset \mathbb{R}^{n}$.

Действительно, пусть $d / 2$ - радиус шара с центром в нуле, который содержит $U$, и пусть $u, v \in U, u \neq v$. Полагая $c=\|u-v\|, a=\langle v, u-v\rangle /\|u-v\|, b=\|v\|^{2}$, имеем

$$
g(t)=\sqrt{(t+a)^{2}+b-a^{2}}, \quad t \in[0, c] .
$$

По неравенству Коши-Буняковского $b-a^{2} \geqslant 0$. Тогда если $b-a^{2}>0$, то функция $g(t)=g_{1}(t)$ СКВ на $[0, c]$ с постоянной $s_{1}(u, v)=1 /(2 \max \{\|u\|,\|v\|\})$ (пример 1$)$. Если $b-a^{2}=0$, то в силу примера $2 g(t)=g_{2}(t)$ CKB на $[0, c]$ с постоянной $s_{2}(u, v)=1 /\|u-v\|$. Поскольку $s_{1}(u, v) \geqslant 1 / d, s_{2}(u, v) \geqslant 1 / d$, то $g(t) \mathrm{CKB} \mathrm{c}$ постоянной $s=1 / d$ на $[0,\|u-v\|], u \neq v$. Итак, по теореме 1 норма СКВ на $U$ с постоянной $s=1 / d$.

Замечание. Евклидова норма не $\mathrm{CB}$ на $U$ (int $U \neq \varnothing)$ и не $\mathrm{CKB}$, если $U-$ неограниченное множество. Другим способом СКВ нормы на ограниченном множестве доказана в [4].

Университет Баньа Лука

Поступило

13.12.94

\section{СПИСОК ЦИТИРОВАННОЙ ЛИТЕРАТУРЫ}

1. Поляк Б. Т. // Докл. АН СССР. 1966. Т. 166. № 2. С. 287-290. 2. Кораблев А. И. // Исслед. по прикл. матем. № 8. Казань, 1980. С. 3-8. 3. Васильев Ф.П. Численные методы решения экстремальных задач. М.: Наука, 1988. 4. Jovanovič M. V. // Radovi Matematički. 1990. V. 6. P. 215-220. 